\title{
Exploratory Study of Sentinel Biomarkers in Gynecologic Cancers
}

Received: February 15, 2018; Accepted: February 20, 2018; Published: February 23, 2018

\section{Early Detection by Sentinel Biomarker in Gynecologic Cancers}

a) Ovarian cancers: In ovarian cancer, even exploratory study of sentinel biomarkers is extremely infant, while detection of sentinel lymphnodes is also difficult and the anatomical regions have been poorly investigated, to predict sentinel nodes. The more accurate lymphatic drainage pathways of the ovaries must be examined. Because metastases of ovarian cancer are lymphatic spread, the sentinel lymphnode can be find out in the para-aortic and paracaval regions, obturator fossa and surrounding internal iliac arteries, and inguinal regions. Thus, the strategy of injecting tracers in both ovarian ligaments to identify sentinel nodes is supported [1]. For ultra-early diagnosis of ovarian cancers, analyses of metaboromes including proteomes and cancer exosomes in serum are of most importance. Especially gene expression within CTCs is investigated frequently. However, studies identifying the clinical utility of these biomarkers have not been reported. Thereby, the additional value for these biomarkers seems to be the investigated evidence levels.

b) Cervical cancers of the uterus: Early detection in cervical cancer can be done by cervical smear test and detecting high-risk HPV. Occult lesions are problematic and cervical conization may be necessary [2].

c) Endometrial cancer of the uterus: In the most cases, endometrial biopsy for early detection of endometrial cancer is necessary although several biopsies may be done for exact diagnosis. Screening method of endometrial cancer by the serum biomarker has not been established. The combination of surface-enhanced laser desorptionionization with bioinformatics tools could help find new biomarkers and establish with high sensitivity and specificity for the detection of endometrial cancer [3]. Involvement of apolipoproteins with endometrial cancer has been suggested as a sentinel biomarker [4].

\section{References}

1 Kleppe M, Kraima AC, Kruitwagen RF, Gorp VT, Smith NN, et al.
Yoshihiro Kikuchi*

Ohki Memorial Kikuchi Cancer Institute for Women, Saitama, Japan

*Corresponding author: Yoshihiro Kikuchi

” QWL04765@nifty.ne.jp

Ohki Memorial Kikuchi Cancer Institute for Women, Saitama, Japan.

Tel: $+81-4-2928-7311$

Citation: Kikuchi Y (2018) Exploratory Study of Sentinel Biomarkers in Gynecologic Cancers. Biomark J. Vol.4 No.1:3

\section{Possibilty of Cancer Exosomes}

Exosomes are a subclass of extracellular vesicles that are released by all cell types, including cancer cells. The cancer exosomes through intracellular communications convey malignant information in the form of proteins, lipids, and nucleic acids that can reprogram recipient cells. Thus, cancer progression and metastases seem to occur through cross-talk of cancer exosome and its micro-environment (cancer stromal cells, fibroblasts and neighboring tissues) [5]. Regarding the metastases, cancer exosomes play crucial roles. Invasion steps of cancer seem to initiate from cross-talking with cancer cells to their neighboring tissues containing cancer stromal tissues by exosomes. Concerning cancer exosomes involvement, exosomes play in specific aspects of tumor progression and metastases containing immune response [6].

\section{Conclusions}

Biomarker research containing cancer exosomes are most important for not only precision medicine (onco-target therapy) but also cancer invasion or/and metastases. Resultantly, improvement of early detection and therapeutic strategies are prospected.

(2015) Understanding lymphatic drainage pathways of the ovaries to predict sites for sentinel nodes in ovarian cancer. Int J Gynecol Cancer 25: 1405-1414. 
2 Sopracordevole F, Clemente N, Alessandrini L, Giuseppe DJ, Cigolot F, et al. (2017) Detection of occult endocervical glandular dysplasia in cervical conization specimens for squamous lesions. Pathol Res Pract 213: $210-216$

3 Zhul LR, Zhang WY, Yu L, Zheng YH, Zhang JZ, et al. (2006) Serum proteomic features for detection of endometrial cancer. Int J Gynecological Cancer 16: 1374-1378.
4 Takano M, Kikuchi Y, Asakawa T, Tomoko G, Tsunekazu K, et al. (2010) Identification of potential serum markers for endometrial cancer using protein expression profiling. J Cancer Res Clin Oncol 136: 475-481.

5 Carolina FR, Barbara A, Miguel S, Sonia AM (2017) The biology of cancer exosomes: Insights and new perspectives. Cancer Res 77: 6480-6488.

6 Hannahan D, Weinberg RA (2011) Hallmarks of cancer: the next generation. Cell 144: 646-674. 